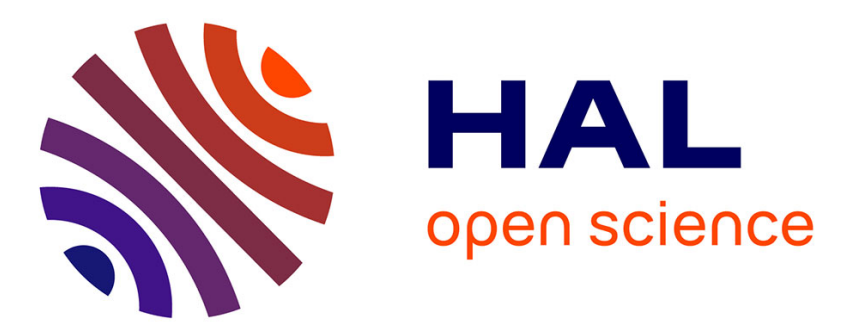

\title{
Magnetic vortices in single crystalline Fe-V disks with four folds magnetic anisotropy
}

K Mitsuzuka, D Lacour, M Hehn, S Andrieu, F Montaigne

\section{To cite this version:}

K Mitsuzuka, D Lacour, M Hehn, S Andrieu, F Montaigne. Magnetic vortices in single crystalline Fe-V disks with four folds magnetic anisotropy. Applied Physics Letters, 2012, 100 (19), pp.192406. 10.1063/1.4711219 . hal-02949024

\section{HAL Id: hal-02949024 \\ https://hal.science/hal-02949024}

Submitted on 25 Sep 2020

HAL is a multi-disciplinary open access archive for the deposit and dissemination of scientific research documents, whether they are published or not. The documents may come from teaching and research institutions in France or abroad, or from public or private research centers.
L'archive ouverte pluridisciplinaire HAL, est destinée au dépôt et à la diffusion de documents scientifiques de niveau recherche, publiés ou non, émanant des établissements d'enseignement et de recherche français ou étrangers, des laboratoires publics ou privés. 


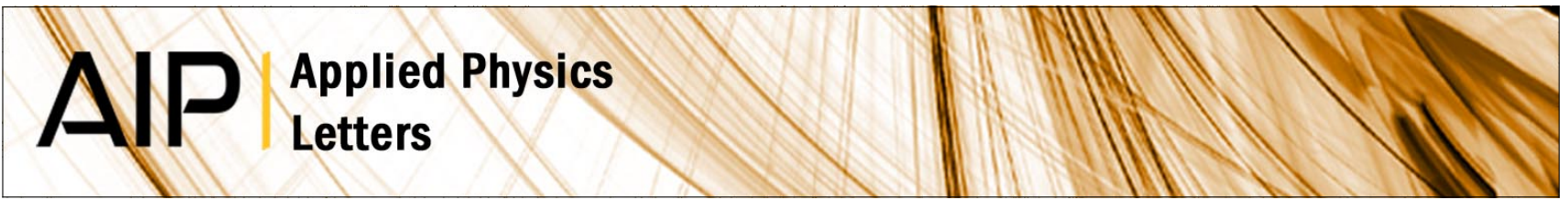

\section{Magnetic vortices in single crystalline Fe-V disks with four folds magnetic anisotropy}

K. Mitsuzuka, D. Lacour, M. Hehn, S. Andrieu, and F. Montaigne

Citation: Appl. Phys. Lett. 100, 192406 (2012); doi: 10.1063/1.4711219

View online: http://dx.doi.org/10.1063/1.4711219

View Table of Contents: http://apl.aip.org/resource/1/APPLAB/v100/i19

Published by the American Institute of Physics.

\section{Related Articles}

Fast domain wall dynamics in MnAs/GaAs films

Appl. Phys. Lett. 101, 072408 (2012)

Sizeable magnetic circular dichroism of artificially precipitated Co clusters in amorphous carbon AlP Advances 2, 032142 (2012)

Non-volatile voltage control of magnetization and magnetic domain walls in magnetostrictive epitaxial thin films Appl. Phys. Lett. 101, 072402 (2012)

Ferromagnetic properties of $\mathrm{GdN}$ thin films studied by temperature dependent circular polarized spectroscopy Appl. Phys. Lett. 101, 072403 (2012)

Influence of intergranular exchange coupling on the magnetization dynamics of CoCrPt:SiO2 granular media J. Appl. Phys. 112, 033918 (2012)

\section{Additional information on Appl. Phys. Lett.}

Journal Homepage: http://apl.aip.org/

Journal Information: http://apl.aip.org/about/about_the_journal

Top downloads: http://apl.aip.org/features/most_downloaded

Information for Authors: http://apl.aip.org/authors

\section{ADVERTISEMENT}

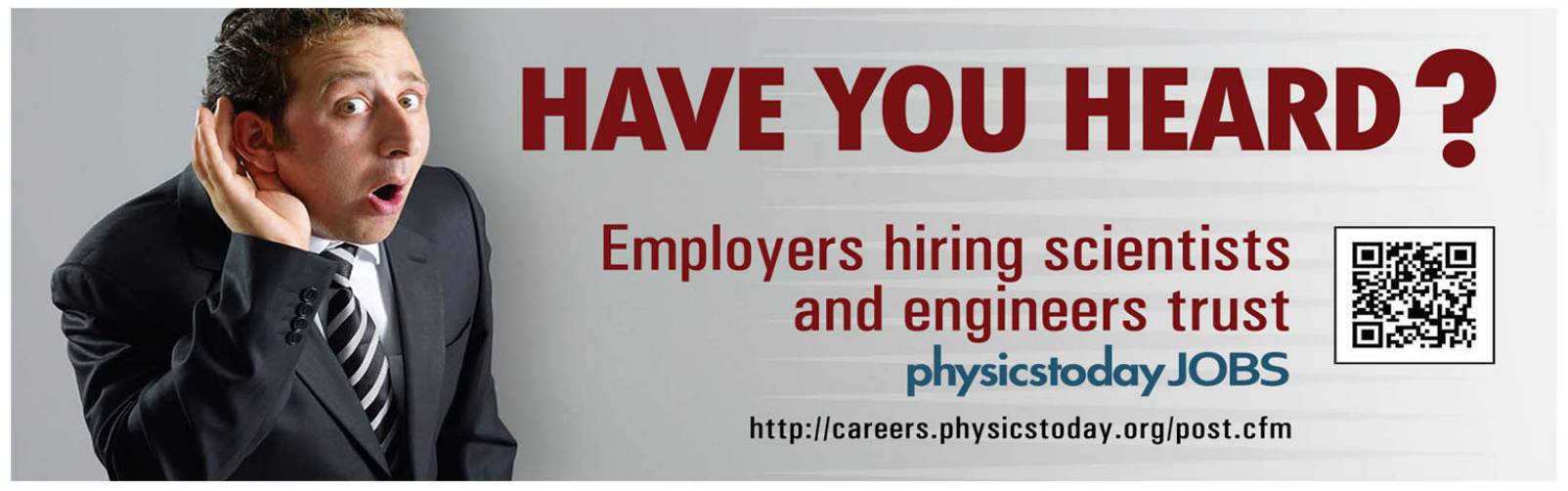




\title{
Magnetic vortices in single crystalline Fe-V disks with four folds magnetic anisotropy
}

\author{
K. Mitsuzuka, D. Lacour, M. Hehn, S. Andrieu, and F. Montaigne \\ Institut Jean Lamour, CNRS - Université de Lorraine, Boulevard des aiguillettes BP 70239 , \\ F-54506 Vandoeuvre lès Nancy, France
}

(Received 3 February 2012; accepted 19 April 2012; published online 8 May 2012)

\begin{abstract}
Magnetic vortices in circular disks made from Fe-V single crystal film have been studied by magnetic force microscopy. At remanent state, all the disks host a single vortex structure. The magnetic contrast differs drastically from the one usually observed in "traditional" materials and reveals the influence of the four folds anisotropy originating from the bcc structure of Fe-V lattice. Field dependence of vortex position has been studied. The vortex displacement is observed to be perpendicular to the field and depends on the field direction. Micromagnetic simulations reproduce quantitatively this behavior and explain the influence of cubic magnetic anisotropy. (C) 2012 American Institute of Physics. [http://dx.doi.org/10.1063/1.4711219]
\end{abstract}

Recently, there is a strong interest in magnetic vortex structures in magnetic disks due to their potentials for attractive applications such as magnetic memory storage devices or spin transfer vortex oscillator (STVO) ${ }^{1-4}$ devices. Indeed, STVO has attractive potentials of high emitted power which is caused by a relatively large vortex core trajectory and narrow linewidth compared with other spin transfer torque oscillators. ${ }^{1,3}$ Ideal material for STVO must combine a low magnetic damping and a high spin polarisation. Epitaxial films of Fe-V alloys have been reported as relatively low magnetic damping materials, with damping parameters ranging between 0.002 and $0.007 . .^{5,6}$ Furthermore, inserted in magnetic tunnel junctions, Fe-V alloys can lead to a significant increase of the tunnel magneto-resistance owing to low misfit between $\mathrm{MgO}$ and $\mathrm{MgO}$ and Fe-V lattice. ${ }^{7,8}$ Then, $\mathrm{Fe}-$ $\mathrm{V}$ based spintronics devices appear to combine all the properties for high performance dynamic applications. However, as pure iron, epitaxial Fe-V films exhibit cubic anisotropy. As a first step, this letter aims at studying the equilibrium configuration of circular disk arrays and highlights the effect of such an anisotropy on the vortex structure in Fe-V disks.

Magnetic disks studied in this letter have been patterned from epitaxial $\mathrm{Fe}-\mathrm{V}$ alloy films. Thin vanadium and iron layers were deposited on $\mathrm{MgO}(100)$ single crystal substrate by molecular beam epitaxy. The Fe and V thicknesses were adjusted to get the proper $\mathrm{V}$ concentration in the alloys and a total thickness of $27 \mathrm{~nm}$. Then these layers were interdiffused by post-annealing process $\left(800^{\circ} \mathrm{C}\right)$. The intermixing process starts at $600{ }^{\circ} \mathrm{C}$ as checked by $\mathrm{x}$-ray photoelectron spectroscopy. An Au capping layer is then deposited to protect the alloy from air. The Fe-V crystallographic structure was confirmed to be bcc using X-ray diffraction. Moreover, since Fe and $\mathrm{V}$ are totally miscible, the (002) lattice spacing of $\mathrm{Fe}-\mathrm{V}$ alloys follows Vegard's law. The determination of this (002) spacing using $\mathrm{x}$-ray diffraction thus allows us to check the $\mathrm{V}$ concentration in the alloy. ${ }^{8}$ For this study, a $10 \% \mathrm{~V}$ concentration was chosen in order to keep large magnetization and anisotropy but with a low damping factor.

The magnetic properties of the full film were studied using vibrating sample magnetometry. Magnetization loops
$(\mathrm{M}(\mathrm{H}))$ along the (100) easy axis (EA) and the (110) hard axis (HA) directions (not shown) confirm the four folds magnetic anisotropy arising from the bcc structure of Fe-V lattice. A saturation magnetization, $M_{\mathrm{s}}$, of $1450 \mathrm{emu} / \mathrm{cm}^{3}$ could be extracted from EA $\mathrm{M}(\mathrm{H})$ loop, a value consistent with a $10 \% \mathrm{~V}$ concentration in the alloy. An anisotropy field, $H_{\mathrm{k}}$, of 500 Oe could be extracted from HA M(H) loop by considering the saturation field of the $\mathrm{M}(\mathrm{H})$ loop leading to an anisotropy constant $K$ of $4.1 \times 10^{5} \mathrm{erg} / \mathrm{cm}^{3}$. The exchange constant of the film, $A$, was evaluated by vector network analyzer ferromagnetic resonance (VNA) FMR measurement as $3 \times 10^{-5} \mathrm{erg} / \mathrm{cm}^{9}$

The Fe-V disks were fabricated using conventional electron beam lithography and Ar ion etching with disk diameters of $1.2,1.65$, and $3.15 \mu \mathrm{m}$. The periodic distance between the disks is twice the diameters of the disks, leading to negligible dipolar fields from neighbour disks. The local micromagnetic configurations of the Fe- $\mathrm{V}$ disks were determined by magnetic force microscopy (MFM) at room temperature. MFM is well known as useful measurement system for observation of magnetic vortex structures. ${ }^{10}$ Furthermore, measurements were done with in-plane applied magnetic field in order to check the position of the vortex core as a function of the applied field direction and intensity.

Figures 1(a)-1(c) show the MFM images measured on 4 disks at remanance. Regardless of the disk diameter, each disk hosts 4 domain walls oriented along the HA making an unconventional cross for a vortex structure. In order to understand this magnetic configuration, 2-dimensional micromagnetic simulations have been performed using the finite difference OOMMF ${ }^{11}$ and GPU MUMAX ${ }^{12}$ micromagnetic codes with the magnetic parameters measured on the Fe-V film. The disk shaped Fe-V was discretized in $4 \times 4 \times 27$ $\mathrm{nm}^{3}$ sized cells, and since the calculation aims at describing the equilibrium position of the vortex core and not its nucleation or its annihilation, thermal effects were not taken into account and the damping parameter has been fixed to 0.5. The calculated magnetic configurations in Fe-V disks are given in Figures 1(d)-1(f) for each disk size. Since magnetization is parallel to the disk edges except at the vortex core, 


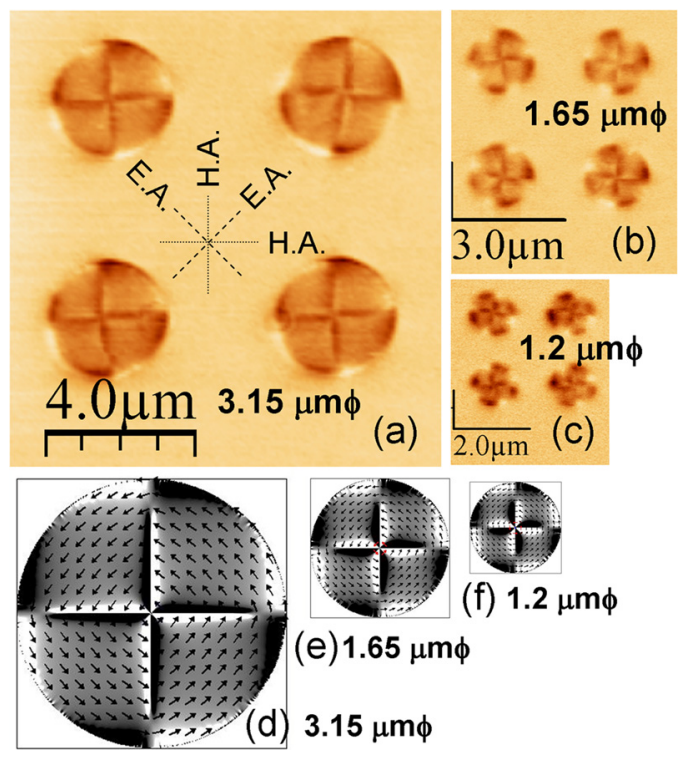

FIG. 1. MFM images of Fe-V disks at zero field with the diameters of (a) 3.15 , (b) 1.65 , and (c) $1.2 \mu \mathrm{m}$. (d)-(f) Computed micromagnetic configurations in a Fe-V disk. (Arrows: direction of magnetization, grey scale contrast: divergence).

the MFM contrast arises from volume magnetic charges given by the divergence of the magnetization. The grey scale divergence/MFM contrast has been superposed to the micromagnetic configuration in Figures 1(d)-1(f). Arrows indicate the direction of magnetization, and the grey scale contrasts indicate the divergence of magnetization. The divergence contrasts at zero field are similar to the MFM images in Figs. 1(a)-1(c). These calculations revealed that every disk has a single vortex structure with core located at the center of the disk. Interestingly, it is clarified that the reason of existence of domain walls in a disk is the existence of 4-fold anisotropy that aligns preferentially the magnetization with the EA directions to decrease the anisotropy energy. It is clear that these vortices have two kinds of chiralities: clockwise (CW) rotation and counter clockwise $(\mathrm{CCW})$ rotation.

The dependence of the magnetic configuration on the magnetic field has then been investigated by applying a tunable field produced by an electromagnet. The field was applied in the plane of the sample and along the EA and HA directions. Figures 2(a) and 2(b) show the MFM images for the $3.15 \mu \mathrm{m} \phi \mathrm{Fe}-\mathrm{V}$ disks with an in-plane applied field of 112 Oe. The vortex cores moved perpendicular to the field direction for both magnetic field directions (EA and HA). The directions of the core motion were opposite depending on the initial chirality of the vortex. Even under applied fields, the MFM contrasts are similar to the calculated divergences from micromagnetic calculations (Figs. 2(c) and 2(d)). The directions of movement of the vortex core in the disk are also reproduced and, as observed, the vortex core always moves perpendicular to the applied field. Micromagnetic calculations revealed that when the magnetic field is applied, the region for which the magnetization is along the field grows, leading to core motion perpendicular to the field. As applied field increased, the core positions moved to the edge of disks in both field directions. In the case of field applied along HA direction, the MFM image shows a complex ripple structure, and the cores are apparently lost at

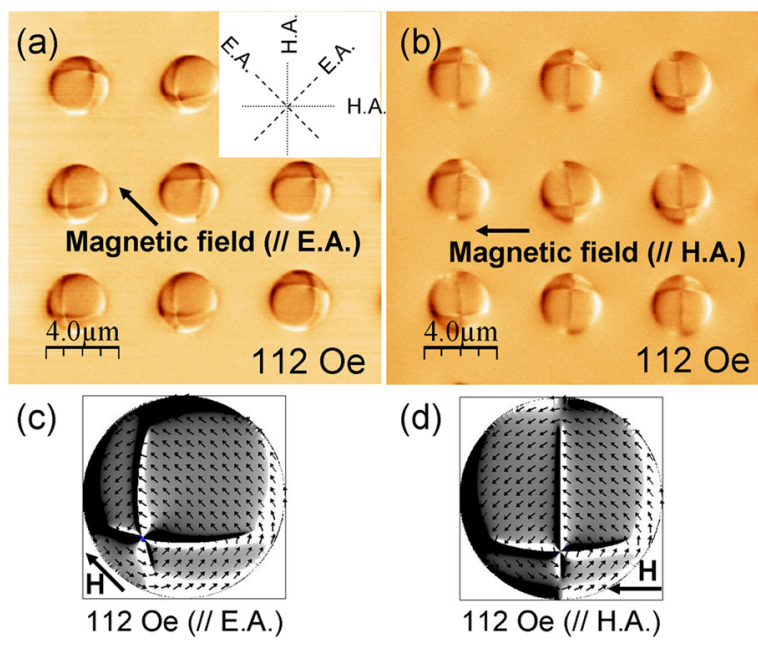

FIG. 2. (a), (b) MFM images of $3.15 \mu \mathrm{m} \phi$ Fe-V disks with in-plane magnetic field (=112 Oe). (c), (d) Calculated magnetic configurations in a Fe-V disk with in-plane magnetic field. (Arrow: direction of magnetization, grey scale contrast: divergence).

310 Oe (Fig. 3). However, micromagnetic simulations show that this ripple structure is still associated to the presence of a vortex (inset of Figure 3) and vanishes when the vortex is annihilated.

In order to show the effect of magnetic anisotropy on $\mathrm{Fe}-\mathrm{V}$ disks, we have measured the core position as a function of applied field, along the EA and HA axes. Figure 4 shows the displacement of the vortex core from the disk center as a function of applied magnetic field for $3.15 \mu \mathrm{m} \phi$ and $1.65 \mu \mathrm{m} \phi$ disks. For the $3.15 \mu \mathrm{m} \phi$ disks, the experimental results are plotted as circular symbols by averaging the values over 9 disks (the standard deviation is about $65 \mathrm{~nm}$ ). At low fields, from 0 to $\sim 100 \mathrm{Oe}$, the position measured experimentally increases linearly with the applied field. For higher fields, the rate of displacement of the vortex with field (susceptibility) decreases, and finally the vortex is expelled from the disk (annihilation). This behavior is similar to the one observed in isotropic materials. ${ }^{13-16}$ For field applied along the EA direction, the annihilation field is $230 \pm 30 \mathrm{Oe}$ (within our field resolution, this field is identical for all disks). It is interesting to note that the vortex core is not driven by the field up to the disk border. The minimum observed distance between vortex cores and disk edge is

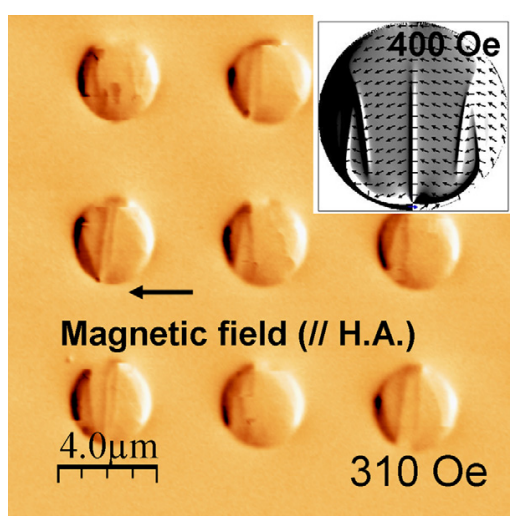

FIG. 3. MFM image of $3.15 \mu \mathrm{m} \phi \mathrm{Fe}-\mathrm{V}$ disks with in-plane magnetic field $(=310 \mathrm{Oe})$. Calculated magnetic configuration in a Fe-V disk with in-plane magnetic field ( $=400 \mathrm{Oe})$ is also shown as inset figure. 


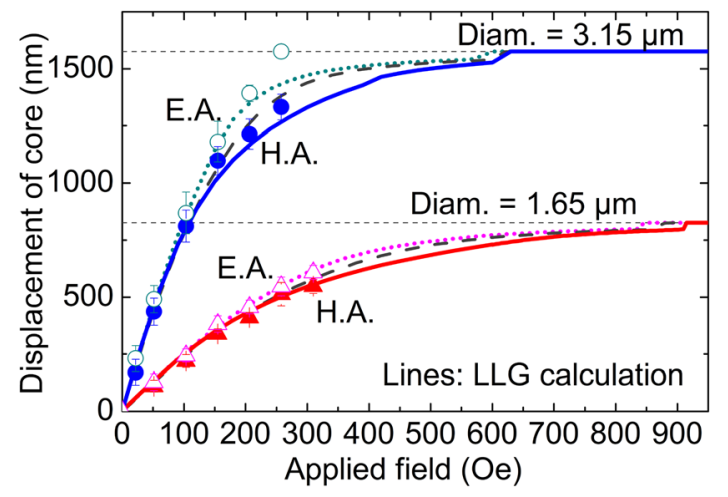

FIG. 4. Displacement of vortex core from the disk center as a function of applied field for 3.15 and $1.65 \mu \mathrm{m} \phi$ disks. Experimental data (averaged values for 9 disks and 8 disks): $3.15 \mu \mathrm{m}$ circular symbols, $1.65 \mu \mathrm{m}$ trianglular symbols; Micomagnetic calculations: lines (solid lines: HA, dot lines: EA, and dashed lines: for isotropic material, $K=0$ ).

$180 \mathrm{~nm}$ just before annihilation. In the case of field applied along HA direction, the displacement of the vortex core is more gradual (lower susceptibility). As mentioned before, the formation of the ripple structure makes it impossible to measure the vortex position for fields above 280 Oe. The limited maximum field of our experimental system does not allow us to image the annihilation of vortices when the field is applied along the hard axis. However, observations of chirality change at remanence suggest an upper bound of 440 Oe for the annihilation field.

In Figure 4, the core position extracted from micromagnetic calculations is also represented as continuous lines. Without any parameter adjustment (all parameters are deduced from independent measurements), a good qualitative agreement between experimental and calculated values of vortex position is obtained for both field directions. However, the value of the experimental annihilation field is significantly lower than the one expected from micromagnetic simulations. The latter is well evidenced in Figure 4 by the abrupt change in vortex position (600 and $630 \mathrm{Oe}$, respectively, for EA and HA). This difference is likely due to thermal effects which are not considered in micromagnetic simulations. Indeed, thermal excitations can make the system to overcome the energy barrier associated to the vortex annihilation. ${ }^{13}$ Annihilation field is thus reduced by temperature. Note that thermal excitations have nearly no influence on vortex core positions in the reversible regime as fluctuations of the vortex are averaged during the observation.

The values of displacements of vortex cores for $1.65 \mu \mathrm{m}$ $\phi$ disks are also shown on Fig. 4 in the same way for $3.15 \mu \mathrm{m} \phi$ disks. The experimental values were obtained by averaging the results for 8 disks. The behavior is analog to the one observed for $3.15 \mu \mathrm{m} \phi$ disks, and a very good agreement between experimental and calculated values is also obtained. However, the annihilation field is higher in these smaller structures. Micromagnetic simulations indicate an annihilation field of, respectively, 845 and 915 Oe along the easy and hard axes which must be compared to 600 and $630 \mathrm{Oe}$ in the $3.15 \mu \mathrm{m} \phi$ disks. Consequently, we have not been able to observe the annihilation of the vortex in the $1.65 \mu \mathrm{m} \phi$ disk due to the limited maximum field of our setup.
Beyond the specific magnetic configurations, the dependence of the vortex position on the direction of the applied field is also a specific feature induced by cubic anisotropy. Thanks to micromagnetic simulations, it is possible to "isolate" the influence of the magnetic anisotropy. Figure 4 represents the theoretical position of the vortex core for a disk without anisotropy (dashed lines). It appears that for a field along the EA, the displacement of the vortex core is increased (the anisotropy reinforces the applied field) whereas it is decreased when the field is applied along the HA (anisotropy competes with the applied field).

From the micromagnetic calculations, the different contributions to the energy can be determined independently. Figure 5 represents both the total energy of the system (square symbols) and its different components as a function of vortex core position for two directions of applied field. As magnetization remains essentially along the two easy axes, the contribution of anisotropy energy is limited. The two main contributions to the total energy are the Zeeman energy (driving force) and the demagnetization energy (restoring force). It is remarkable that for a given core distance from the center, demagnetization energy is the same as the vortex core is moving parallel to the EA or HA. The restoring force is then isotropic as for material without magnetic anisotropy. On the other hand, the Zeeman energy depends on the vortex displacement direction due to the in-plane magnetic anisotropy.

In this study, we have investigated the magnetic vortex structures of Fe-V epitaxial disks using a dedicated MFM set-up and micromagnetic simulations using the magnetic parameters of the film determined experimentally. It revealed that all of the Fe-V disks have a single vortex structure with a single core and typical 4 domain walls. This peculiar behavior strongly differs from that observed in usually used materials like permalloy and is explained taking into account the in-plane 4-folds magnetic anisotropy observed in these alloys. The mechanism of vortex core motion with the applied field determined by MFM is well understood with the help of simulations. We have thus demonstrated that the movement of the vortex core can be controlled by tuning the in-plane magnetic field direction and intensity. Such control of the vortex configuration as well as the low damping in

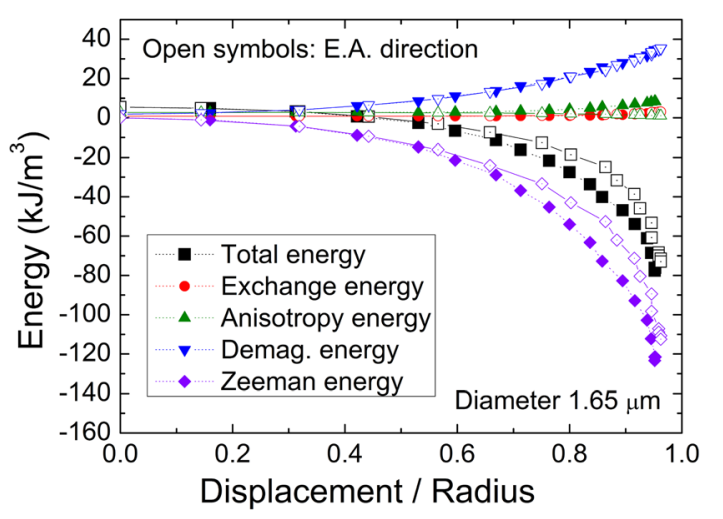

FIG. 5. Total energy and its different components as a function of normalized core position for a $1.65 \mu \mathrm{m} \phi$ disk when the field is applied parallel to the hard axis (solid symbols) and parallel to the easy axis (open symbols). 
these Fe-V alloys are key-points to deeply understand the physics involved in spin transfer vortex oscillators.

This work has been partially supported by the ANR Grant VOICE (ANR-09-NANO-006). The authors acknowledge support from G. Lengaigne and S. Mc Murtry for the nanofabrication process, S. Robert for X-ray diffraction measurements, and T. Devolder and R. Otxoa for VNAFMR measurements and fruitful discussions.

${ }^{1}$ A. Dussaux, B. Georges, J. Grollier, V. Cros, A. V. Khvalkovskiy, A. Fukushima, M. Konoto, H. Kubota, K. Yakushiji, S. Yuasa, K. A. Zvezdin, K. Ando, and A. Fert, Nature Commun. 1, 8 (2010).

${ }^{2}$ A. V. Khvalkovskiy, J. Grollier, A. Dussaux, K. A. Zvezdin, and V. Cros, Phys. Rev. B 80, 140401 (2009).

${ }^{3}$ N. Locatelli, V. V. Naletov, J. Grollier, G. de Loubens, V. Cros, C. Deranlot, C. Ulysse, G. Faini, O. Klein, and A. Fert, Appl. Phys. Lett. 98, 062501 (2011).

${ }^{4}$ T. Devolder, J. V. Kim, S. Petit-Watelot, R. Otxoa, C. Chappert, M. Manfrini, W. Van Roy, and L. Lagae, IEEE Trans. Magn. 47, 1595 (2011).

${ }^{5}$ C. Scheck, L. Cheng, I. Barsukov, Z. Frait, and W. E. Bailey, Phys. Rev. Lett. 98, 117601 (2007).
${ }^{6}$ J.-M. L. Beaujour, A. D. Kent, D. W. Abraham, and J. Z. Sun, J. Appl. Phys. 103, 07B519 (2008).

${ }^{7}$ F. Bonell, S. Andrieu, F. Bertran, P. Lefèvre, A. T. Ibrahimi, E. Snoeck, C. Tiusan, and F. Montaigne, IEEE Trans. Magn. 45, 3467 (2009).

${ }^{8}$ F. Bonell, S. Andrieu, C. Tiusan, F. Montaigne, E. Snoeck, B. Belhadji, L. Calmels, F. Bertran, P. Le Fèvre, and A. Taleb-Ibrahimi, Phys. Rev. B 82, 092405 (2010).

9"VNA-FMR method" (unpublished); G. Counil, J.-V. Kim, T. Devolder, P. Crozat, C. Chappert, and A. Cebollada, J. Appl. Phys. 98, 023901 (2005).

${ }^{10}$ T. Shinjo, T. Okuno, R. Hassdorf, K. Shigeto, and T. Ono, Science 289, 930 (2000).

${ }^{11}$ M. J. Donahue and D. G. Porter, OOMMF User's Guide Version 1.0," NISTIR 6376 (National Institute of Standards and Technology, Gaithersburg, MD, 1999).

${ }^{12}$ A. Vansteenkiste and B. Van de Wiele, J. Magn. Magn. Mater. 323, 2585 (2011).

${ }^{13}$ K. Yu. Guslienko, V. Novosad, Y. Otani, H. Shima, and K. Fukamichi, Appl. Phys. Lett. 78, 3848 (2001).

${ }^{14}$ K. Yu. Guslienko, V. Novosad, Y. Otani, H. Shima, and K. Fukamichi, Phys. Rev. B 65, 024414 (2001).

${ }^{15}$ S. R. Bakaul, B. L. Wu, G. C. Han, and Y. H. Wu, Appl. Phys. Lett. 97, 042503 (2010).

${ }^{16}$ G. N. Kakazei, M. Ilyn, O. Chubykalo-Fesenko, J. Gonzalez, A. A. Serga, A. V. Chumak, P. A. Beck, B. Laegel, B. Hilebrands, and K. Y. Guslienko, Appl. Phys. Lett. 99, 052512 (2011). 